\title{
Effect of treatment modality on in-hospital outcome in patients with subarachnoid hemorrhage: a nationwide study in Japan (J-ASPECT Study)
}

\author{
${ }^{*}$ Ryota Kurogi, MD, ${ }^{1}$ Akiko Kada, MPH, ${ }^{2}$ Kunihiro Nishimura, MD, PhD, ${ }^{3}$ Satoru Kamitani, MD, ${ }^{4}$ \\ Ataru Nishimura, MD, ${ }^{1}$ Tetsuro Sayama, MD, PhD, ${ }^{1}$ Jyoji Nakagawara, MD, PhD, ${ }^{5}$ \\ Kazunori Toyoda, MD, PhD, ${ }^{6}$ Kuniaki Ogasawara, MD, PhD, ${ }^{7}$ Junichi Ono, MD, PhD, ${ }^{8}$ \\ Yoshiaki Shiokawa, MD, PhD, ${ }^{9}$ Toru Aruga, MD, PhD, ${ }^{10}$ Shigeru Miyachi, MD, PhD, ${ }^{11}$ \\ Izumi Nagata, MD, PhD,12 Shinya Matsuda, MD, PhD, ${ }^{13}$ Shinichi Yoshimura, MD, PhD, ${ }^{14}$ \\ Kazuo Okuchi, MD, PhD, ${ }^{15}$ Akifumi Suzuki, MD, PhD, ${ }^{16}$ Fumiaki Nakamura, MD, PhD, ${ }^{4}$ \\ Daisuke Onozuka, PhD, ${ }^{17}$ Akihito Hagihara, DMSc, MPH, ${ }^{17}$ Koji lihara, MD, PhD, ${ }^{1}$ and \\ the J-ASPECT Study Collaborators
}

\begin{abstract}
${ }^{1}$ Department of Neurosurgery, Graduate School of Medical Sciences, Kyushu University, Fukuoka;, 'Department of Clinical Trials and Research, National Hospital Organization Nagoya Medical Centre, Nagoya; ${ }^{3}$ Department of Preventive Medicine and Epidemiologic Informatics, National Cerebral and Cardiovascular Centre, Suita; ${ }^{4}$ Department of Public Health/Health Policy, Graduate School of Medicine, University of Tokyo; ${ }^{5}$ Integrative Stroke Imaging Centre; ${ }^{6}$ Department of Cerebrovascular Medicine, National Cerebral and Cardiovascular Centre, Suita; ${ }^{7}$ Department of Neurosurgery, Iwate Medical University, Morioka; ${ }^{8}$ Department of Neurosurgery, Chiba Cerebral and Cardiovascular Centre, Chiba; ${ }^{9}$ Department of Neurosurgery, Kyorin University, Mitaka; ${ }^{10}$ Department of Emergency and Critical Care Medicine, Showa University Hospital, Shinagawa; ${ }^{11}$ Department of Neurosurgery, Osaka Medical College, Takatsuki; ${ }^{12}$ Department of Neurosurgery, Kokura Memorial Hospital, Kitakyushu; ${ }^{13}$ Department of Preventive Medicine and Community Health, School of Medicine, University of Occupational and Environmental Health, Kitakyushu; ${ }^{14}$ Department of Neurosurgery, Hyogo College of Medicine, Nishinomiya; ${ }^{15}$ Department of Emergency and Critical Care Medicine, Nara Medical University, Kashihara; ${ }^{16}$ Department of Surgical Neurology, Research Institute for Brain and Blood Vessels-Akita; and ${ }^{17}$ Department of Health Communication, Graduate School of Medical Sciences, Kyushu University, Fukuoka, Japan
\end{abstract}

OBJECTIVE Although heterogeneity in patient outcomes following subarachnoid hemorrhage (SAH) has been observed across different centers, the relative merits of clipping and coiling for SAH remain unknown. The authors sought to compare the patient outcomes between these therapeutic modalities using a large nationwide discharge database encompassing hospitals with different comprehensive stroke center (CSC) capabilities.

METHODS They analyzed data from 5214 patients with SAH (clipping 3624, coiling 1590) who had been urgently hospitalized at 393 institutions in Japan in the period from April 2012 to March 2013. In-hospital mortality, modified Rankin Scale (mRS) score, cerebral infarction, complications, hospital length of stay, and medical costs were compared between the clipping and coiling groups after adjustment for patient-level and hospital-level characteristics by using mixedmodel analysis.

RESULTS Patients who had undergone coiling had significantly higher in-hospital mortality ( $12.4 \%$ vs $8.7 \%$, OR 1.3 ) and a shorter median hospital stay (32.0 vs 37.0 days, $p<0.001$ ) than those who had undergone clipping. The respective proportions of patients discharged with mRS scores of 3-6 (46.4\% and 42.9\%) and median medical costs (thousands US\$, 35.7 and 36.7) were not significantly different between the groups. These results remained robust after further adjustment for CSC capabilities as a hospital-related covariate.

ABBREVIATIONS BRAT = Barrow Ruptured Aneurysm Trial; $\mathrm{CCl}=$ Charlson Comorbidity Index; $\mathrm{CSC}=$ comprehensive stroke center; $\mathrm{DPC}=$ Diagnosis Procedure Combination; HAC = hospital-acquired condition; ISAT = International Subarachnoid Aneurysm Trial; JCS = Japan Coma Scale; mRS = modified Rankin Scale; PPV = positive predictive value; $\mathrm{PSI}=$ patient safety indicator; $\mathrm{RCT}=$ randomized controlled trial; $\mathrm{SAH}=$ subarachnoid hemorrhage .

SUBMITTED April 22, 2016. ACCEPTED December 9, 2016.

INCLUDE WHEN CITING Published online May 26, 2017; DOI: 10.3171/2016.12.JNS161039.

* Dr. Kurogi and Ms. Kada contributed equally to this work. 
CONCLUSIONS Despite the increasing use of coiling, clipping remains the mainstay treatment for SAH. Regardless of CSC capabilities, clipping was associated with reduced in-hospital mortality, similar unfavorable functional outcomes and medical costs, and a longer hospital stay as compared with coiling in 2012 in Japan. Further study is required to determine the influence of unmeasured confounders.

https://thejns.org/doi/abs/10.3171/2016.12.JNS161039

KEY WORDS subarachnoid hemorrhage; clipping; coiling; nationwide database study; comprehensive stroke center; vascular disorders

$\mathrm{N}$ UMEROUS studies have compared outcomes between clipping and coiling procedures for patients with subarachnoid hemorrhage (SAH) ${ }^{7,17-21,26,28,30,31}$ The International Subarachnoid Aneurysm Trial (ISAT), a large randomized controlled trial (RCT) that compared neurosurgical clipping with endovascular coiling in patients with $\mathrm{SAH}$, demonstrated the superiority of coiling. ${ }^{20}$ This finding of the ISAT led to a prominent shift in the pattern of treatment for $\mathrm{SAH}$, with the number of coiling procedures steadily increasing following publication. ${ }^{28}$ However, it remains questionable whether the results of the ISAT can be generalized to all patients with SAH because most participants enrolled in the study were high-clinicalgrade patients with small anterior circulation aneurysms. ${ }^{23}$ While patient demographics and SAH severity remain the strongest predictors of outcome following treatment, interhospital, interregional, and cross-national disparities may also contribute to differences in patient outcomes. For example, fatality rates in patients with SAH in Japan are substantially lower than those in other regions. ${ }^{24}$ Such differences may be explained by the varied expertise of the treating physician, the SAH case volumes at different institutions, and a hospital's propensity to use endovascular therapy. ${ }^{3,11,13}$ Recently, using the Japanese Diagnosis Procedure Combination (DPC) database, we established that high comprehensive stroke center (CSC) capability scores were associated with reduced in-hospital mortality in patients with ischemia and hemorrhage..$^{15}$

Researchers have increasingly relied on the use of administrative databases of hospital admissions to assess clinical outcomes. Despite several limitations, studies using nationwide databases produce more generalizable results than those of RCTs because of the elimination of potential selection and referral biases.,17,26 Previous large database studies that have compared outcomes following clipping and coiling in patients with SAH have been conducted in the US, yielding mixed results. ${ }^{7,17}$

To address these discrepancies, we used the nationwide DPC database (JASPECT Study) to identify patients urgently hospitalized for SAH between April 1, 2012, and March 31, 2013. We then performed a mixed-model analysis adjusted with patient-level and hospital-level covariates to compare outcomes for patients with SAH who had undergone clipping and coiling procedures.

\section{Methods \\ The DPC Database}

The DPC database is a mixed-case classification sys- tem linked with a lump-sum payment system that was launched in 2002 by the Ministry of Health, Labor, and Welfare of Japan. ${ }^{34}$ In 2010, an estimated 1388 acute care hospitals, representing about $50 \%$ of all hospital beds, adopted the DPC system. Data on practices can be obtained from the DPC database, and the attending physician is responsible for clinical data entry for each patient. The DPC system includes all patients admitted to participating hospitals. Compared with other registry databases, the DPC database has strength in enabling researchers to conduct nationwide studies of descriptive or analytical epidemiology in the real-world setting of clinical practice. The Japanese DPC database includes data on the following elements: each patient's profile (that is, age, sex, height, weight, smoking index); principal diagnoses (coded according to the International Classification of Diseases and Injuries, 10th revision); comorbidities at admission (coded similarly); complications after admission (coded similarly); and procedures, including surgery, medications, and devices used during hospitalization; length of stay; discharge status; and medical expense..$^{2,34,35}$ Institutions using the DPC system encompass a wide variety of centers, including academic, large, urban, and rural hospitals. ${ }^{15}$ Of the 847 certified training institutions of the Japan Neurosurgical Society, 393 agreed to participate in the J-ASPECT Study. This cross-sectional survey used DPC discharge data from the participating institutions.

\section{Assessment of CSC Capabilities}

We investigated whether the effect of treatment modality on outcomes in patients with $\mathrm{SAH}$ was influenced by CSC capabilities. The CSC capabilities questionnaire was completed by 266 of the 393 participating institutions. This institutional survey has been described in detail elsewhere. ${ }^{15}$ The CSC capabilities were assessed using 25 items specifically recommended for $\mathrm{CSC},{ }^{1}$ which were divided into 5 categories: 1) personnel (7 items: boardcertified neurologists, board-certified neurosurgeons, board-certified endovascular physicians, board-certified physicians in critical care medicine, board-certified physicians in physical medicine and rehabilitation, personnel in rehabilitation therapy, and stroke rehabilitation nurses); 2) diagnostic techniques (6 items: 24 hours/day, 7 days/week [24/7] availability of CT, MRI with diffusion-weighted imaging, digital cerebral angiography, CT angiography, carotid duplex ultrasound, and transcranial Doppler ultrasonography); 3) specific expertise (5 items: carotid endarterectomy, clipping of intracranial aneurysms [IAs], re- 
moval of intracerebral hemorrhage, coiling of IAs, and intraarterial reperfusion therapy); 4) infrastructure (5 items: stroke unit, intensive care unit, operating room staffed 24/7, interventional services coverage 24/7, and stroke registry); and 5) educational components (2 items: community education and professional education). One point was assigned for each recommended item, with a maximum possible CSC score of 25 .

\section{Sampling Strategy}

Computer software was developed to identify patients hospitalized for SAH from the de-identified discharge database using the ICD-10 diagnosis codes related to SAH (I60.0-9). We extracted data from the Japanese DPC database for patients who had been urgently hospitalized for SAH in the period from April 1, 2012, to March 31, 2013. Patients with a scheduled admission were excluded from our study.

The DPC database includes the following data: hospitals' unique identifiers and CSC scores; patients' age, sex, height, and weight; diagnoses; comorbidities on admission, including those based on the Charlson Comorbidity Index (CCI);6 Brinkman Index $;{ }^{4}$ level of consciousness on admission according to the Japan Coma Scale (JCS); admission source; days between admission and treatment; inhospital mortality; modified Rankin Scale (mRS) score at discharge; cerebral infarction after admission; complications after admission according to patient safety indicators (PSIs) and hospital-acquired conditions (HACs) ${ }^{10}$ length of hospital stay; medical costs; and procedures coded with Japanese original K-codes. The extent of smoking was assessed based on the Brinkman Index (daily number of cigarettes $\times$ years). ${ }^{4}$ The JCS is the most widely used grading scale for assessing impaired consciousness in Japan (Supplementary Table S1). ${ }^{14,29}$ This scale represented levels of consciousness upon admission regardless of whether patients had subsequently undergone ventriculostomy or CSF shunt placement.

\section{Validation Study}

We collected a $1 \%$ representative sample from among 501,609 patients at the 393 institutions included in the DPC discharge database from April 1, 2012, to March 31,2013 , by using 2 -stage random sampling. Admissions were randomly drawn from among the 393 included institutions, after which patients with SAH were randomly selected using ICD-10 codes. The primary diagnosis, treatment modality, in-hospital mortality, mRS score at discharge, and JCS score on admission were verified based on the discharge summary, hospital records of the clinical presentation according to neurosurgeons or neurologists, and CT or MRI evidence. Validation of the attending physician's contemporary assessment was equivalent to that of a retrospective chart review. The sensitivity, specificity, and positive predictive value (PPV) of SAH as the primary diagnosis were calculated using the sample set. The sensitivities, specificities, and PPVs of treatment modality, in-hospital mortality, mRS score at discharge, and JCS score on admission were calculated using the ICD-10 diagnosis codes related to SAH (I60.0-9) in the sample set.

\section{Statistical Analysis}

For statistical analysis, the mRS score at discharge was dichotomized into score groups $0-2$ and 3-6. The JCS score was treated as a categorical variable of $0,1,2$, or 3 . Differences in patient demographics between the clipping and coiling groups were analyzed using the Wilcoxon rank-sum and chi-square tests. We used mixed-model analysis to estimate odds ratios for in-hospital mortality, $\mathrm{mRS}$ score at discharge, cerebral infarction, and complications and to estimate differences in length of hospital stay and medical costs. Odds ratios and differences were adjusted using a mixed model with sex, age, JCS score, and CCI score as fixed effects and hospital as a random effect (full cohort). To examine whether patient outcomes were affected by the CSC capabilities of each institution, the CSC score was applied as a fixed effect in addition to the aforementioned variables (subgroup with CSC scores). After conducting stratified analysis by JCS 0, 1-digit, 2-digit, and 3-digit codes, odds ratios and differences were adjusted using a mixed model with sex, age, CCI scores, and treatment modalities (clipping or coiling) as fixed effects and hospital as a random effect. We estimated odds ratios and differences for outcomes after 1:1 propensity score matching to account for differences in baseline characteristics between groups. Propensity scores for coiling were estimated using a probit model with age, sex, JCS score, and CCI score. To match patients, we used an automated matching procedure in the STATA software package that randomly selected a patient with clipping and a patient with coiling from the pool of potential patients within a propensity score caliper of \pm 0.001 . Successfully matched pairs were removed, and the procedure was repeated until all patients were matched to one comparator or until no further patients that could be matched were available within the caliper. Analyses were performed using SAS version 9.3 (SAS Institute Inc.), STATA version 12 (STATA Corp.), and SPSS version 12 (SPSS Inc.). A p value < 0.05 was considered statistically significant.

\section{Ethics Statement}

This research was designed by us and approved by the institutional review board of Kyushu University, which waived the requirement for individual informed consent.

\section{Results}

\section{Patient Demographics}

A total of 5214 patients with SAH (clipping 3624, coiling 1590) at 393 institutions were identified based on ICD10 codes (full cohort). Of the 4327 patients $(83 \%)$ with SAH who were hospitalized at the 266 institutions that participated in the CSC capabilities survey and assigned a CSC score (subgroup with CSC scores), 2996 had undergone clipping and 1331 had undergone coiling. Table 1 shows the demographics of the full cohort and the subgroup with CSC scores.

In the full cohort, significant differences in some characteristics were observed between patients who had undergone clipping and those who had undergone coiling. Specifically, patients who underwent coiling were significantly older (mean age: clipping 62.0 years, coiling 63.5 
TABLE 1. Demographics of the full cohort and the subgroup with CSC scores

\begin{tabular}{|c|c|c|c|c|c|c|}
\hline \multirow[b]{2}{*}{ Parameter } & \multicolumn{3}{|c|}{ Full Cohort } & \multicolumn{3}{|c|}{ Subgroup w/ CSC Scores } \\
\hline & Clipping & Coiling & $p$ Value & Clipping & Coiling & $p$ Value \\
\hline Total & 3624 & 1590 & & 2996 & 1331 & \\
\hline \multicolumn{7}{|l|}{ Patients } \\
\hline Male & $1147(31.6)$ & $480(30.2)$ & 0.294 & $955(31.9)$ & $388(29.2)$ & 0.074 \\
\hline Age in yrs & $62.0 \pm 13.9$ & $63.5 \pm 15.3$ & 0.003 & $62.1 \pm 13.9$ & $63.3 \pm 15.3$ & 0.025 \\
\hline Height in $\mathrm{cm}$ & $157.9 \pm 9.2$ & $157.4 \pm 9.6$ & 0.080 & $157.9 \pm 9.2$ & $157.3 \pm 9.6$ & 0.066 \\
\hline Weight in kg & $57 \pm 12.4$ & $56.2 \pm 13.3$ & 0.005 & $57.0 \pm 12.5$ & $56.1 \pm 13.3$ & 0.004 \\
\hline Brinkman Index & $215.4 \pm 376.7$ & $164.9 \pm 387.4$ & $<0.001$ & $220.5 \pm 384.7$ & $158.4 \pm 351.5$ & $<0.001$ \\
\hline \multicolumn{7}{|l|}{ Comorbidities } \\
\hline CCI score & $4.0 \pm 1.0$ & $4.1 \pm 1.6$ & 0.034 & $4.0 \pm 1.5$ & $4.1 \pm 1.6$ & 0.166 \\
\hline Hypertension & $1993(55.0)$ & $852(53.6)$ & 0.347 & $1658(55.3)$ & 709 (53.3) & 0.206 \\
\hline Diabetes mellitus & $352(9.7)$ & $133(8.4)$ & 0.123 & $278(9.3)$ & $109(8.2)$ & 0.246 \\
\hline Hyperlipidemia & $565(15.6)$ & $236(14.8)$ & 0.491 & $455(15.2)$ & $190(14.3)$ & 0.437 \\
\hline Hyperuricemia & $12(0.3)$ & $9(0.6)$ & 0.218 & $11(0.4)$ & $8(0.6)$ & 0.283 \\
\hline JCS score on admission & & & $<0.001$ & & & $<0.001$ \\
\hline 0 & $834(23.0)$ & $285(17.9)$ & & $663(22.1)$ & $245(18.4)$ & \\
\hline 1-digit code & $1120(30.9)$ & $450(28.3)$ & & $951(31.7)$ & $371(27.9)$ & \\
\hline 2-digit code & $811(22.4)$ & $363(22.8)$ & & $673(22.5)$ & $320(24.0)$ & \\
\hline 3-digit code & $859(23.7)$ & $492(30.9)$ & & $709(23.7)$ & $395(29.7)$ & \\
\hline \multicolumn{7}{|l|}{ Admission source } \\
\hline Ambulance & $2837(78.3)$ & $1244(78.2)$ & 0.971 & $2369(79.1)$ & $1045(78.5)$ & 0.671 \\
\hline Days btwn admission \& treatment & $2.0 \pm 3.3$ & $2.0 \pm 4.1$ & 0.141 & $2.0 \pm 3.4$ & $2.0 \pm 3.7$ & 0.076 \\
\hline \multicolumn{7}{|l|}{ Surgery for hydrocephalus } \\
\hline Ventriculostomy & $182(5.0)$ & $322(20.3)$ & $<0.001$ & $160(5.3)$ & $267(20.1)$ & $<0.001$ \\
\hline CSF shunt & $802(22.1)$ & $270(17.0)$ & $<0.001$ & $682(22.8)$ & $225(16.9)$ & $<0.001$ \\
\hline \multicolumn{7}{|l|}{ Hospital characteristics (CSC scores) } \\
\hline Total score & NA & NA & NA & $16.8 \pm 3.6$ & $17.9 \pm 2.8$ & $<0.001$ \\
\hline Personnel & NA & NA & NA & $3.9 \pm 1.3$ & $4.2 \pm 1.1$ & $<0.001$ \\
\hline Diagnostic techniques & NA & NA & NA & $4.4 \pm 1.0$ & $4.4 \pm 1.0$ & 0.286 \\
\hline Specific expertise & NA & NA & NA & $4.3 \pm 0.9$ & $4.8 \pm 0.6$ & $<0.001$ \\
\hline Infrastructure & NA & NA & NA & $2.8 \pm 1.4$ & $3.2 \pm 1.3$ & $<0.001$ \\
\hline Educational components & NA & NA & NA & $1.3 \pm 0.8$ & $1.3 \pm 0.8$ & 0.971 \\
\hline
\end{tabular}

$\mathrm{NA}=$ not applicable.

Values expressed as the mean \pm standard deviation or number (\%).

years, $\mathrm{p}=0.003$ ). The proportion of patients with impaired consciousness was significantly higher in the coiling group (for example, comatose patients: clipping $23.7 \%$, coiling $30.9 \%, \mathrm{p}<0.001)$. The mean CCI score was also significantly higher in the coiling group (clipping $4.0 \pm$ 1.0 , coiling $4.1 \pm 1.6, p=0.034$ ). No significant differences in other comorbidities, such as hypertension, diabetes mellitus, hyperlipidemia, and hyperuricemia, were noted between the groups. The mean weight (clipping 57.0 $\pm 12.4 \mathrm{~kg}$, coiling $56.2 \pm 13.3 \mathrm{~kg}, \mathrm{p}=0.005$ ) and mean Brinkman Index (clipping $215.4 \pm 376.7$, coiling $164.9 \pm$ $387.4, \mathrm{p}<0.001)$ were significantly lower in the coiling group.

In the subgroup with CSC scores, the corresponding differences between the 2 treatment groups were also significant except in the mean CCI score (clipping $4.0 \pm 1.5$, coiling $4.1 \pm 1.6, \mathrm{p}=0.166)$. Regarding hospital charac- teristics, the mean CSC total (clipping $16.8 \pm 3.6$, coiling $17.9 \pm 2.8, \mathrm{p}<0.001$ ), personnel (clipping $3.9 \pm 1.3$, coiling $4.2 \pm 1.1, \mathrm{p}<0.001$ ), specific expertise (clipping $4.3 \pm 0.9$, coiling $4.8 \pm 0.6, \mathrm{p}<0.001$ ), and infrastructure subgroup scores (clipping $2.8 \pm 1.4$, coiling $3.2 \pm 1.3, \mathrm{p}<0.001$ ) were significantly higher in the coiling group.

\section{Mixed-Model Analysis}

Table 2 shows outcomes in the full cohort and the subgroup with CSC scores. In the full cohort, rates of in-hospital mortality (clipping 8.7\%, coiling $12.4 \%$ ), cerebral infarction (clipping 16.7\%, coiling 18.1\%), mRS score of 3-6 at discharge (clipping 42.9\%, coiling 46.4\%), and PSIs $\geq 1$ (clipping 48.5\%, coiling 63.1\%) were higher in the coiling group. However, the proportion of HACs $\geq 1$ was higher in the clipping group (7.9\% vs $7.0 \%$ ). The median hospital 
TABLE 2. Outcomes in the full cohort and the subgroup with CSC scores

\begin{tabular}{|c|c|c|c|c|}
\hline \multirow[b]{2}{*}{ Parameter } & \multicolumn{2}{|c|}{ Full Cohort } & \multicolumn{2}{|c|}{ Subgroup w/ CSC Scores } \\
\hline & Clipping & Coiling & Clipping & Coiling \\
\hline Total & 3624 & 1590 & 2996 & 1331 \\
\hline In-hospital mortality & $315(8.7)$ & $198(12.4)$ & $257(8.6)$ & $152(11.4)$ \\
\hline Discharge mRS Scores 3-6 & $1555(42.9)$ & $738(46.4)$ & $1307(43.6)$ & $618(46.4)$ \\
\hline Cerebral infarction & $607(16.7)$ & $288(18.1)$ & $500(16.7)$ & $237(17.8)$ \\
\hline PSIs $\geq 1$ & $1757(48.5)$ & $1003(63.1)$ & $1442(48.1)$ & $856(64.3)$ \\
\hline $\mathrm{HACs} \geq 1$ & $288(7.9)$ & $111(7.0)$ & $240(8.0)$ & $97(7.3)$ \\
\hline Length of hospital stay in days & $37.0 \pm 33.7$ & $32.0 \pm 30.7$ & $38.0 \pm 33.9$ & $32.0 \pm 30.7$ \\
\hline Medical costs in thousands US\$ & $36.7 \pm 14.1$ & $35.7 \pm 14.9$ & $36.7 \pm 14.1$ & $36.0 \pm 14.5$ \\
\hline
\end{tabular}

Values expressed as the median \pm standard deviation or number (\%).

stay was longer in the clipping group (median days: clipping 37.0, coiling 32.0), whereas the median medical costs were similar between the groups (thousands US\$: clipping 36.7, coiling 35.7). Similar results were obtained for the subgroup with CSC scores.

Table 3 shows patient outcomes in the full cohort and the subgroup with CSC scores according to the mixedmodel analysis. In the full cohort, after adjusting for sex, age, JCS and CCI scores as fixed effects and hospital as a random effect, in-hospital mortality was significantly higher in the coiling group (OR 1.30, $\mathrm{p}=0.013)$. Rates of cerebral infarction (OR 1.19, $\mathrm{p}=0.066)$ and $\mathrm{mRS}$ scores of $3-6$ at discharge (OR 0.98, $\mathrm{p}=0.761$ ) were not significantly different between the treatment groups. The proportion of PSIs $\geq 1$ was significantly higher in the coiling group (OR 1.3, p $=0.003$ ), whereas that of HACs $\geq 1$ was significantly higher in the clipping group (OR $0.73, \mathrm{p}=0.021$ ). The median hospital stay was significantly longer in the clipping group $(\beta=-7.18, p<0.001)$, whereas the medical costs were not significantly different between the groups $(\beta=-144.64, p=0.742)$. Analysis of the subgroup with CSC scores revealed similar results regarding in-hospital mortality (OR 1.27, $\mathrm{p}=0.046)$, cerebral infarction (OR $1.18, \mathrm{p}=0.124), \mathrm{mRS}$ scores of $3-6$ at discharge (OR 0.96, $p=0.657)$, PSIs $\geq 1$ (OR 1.33, $p=0.003)$, length of hospital stay $(\beta=-6.98, p<0.001)$, and medical costs $(\beta=$ $-603.15, p=0.210$ ). Although the proportion of HACs $\geq 1$ was no longer significantly different between the groups, the odds ratio exhibited a similar tendency to that in the full cohort (OR 0.78, $\mathrm{p}=0.105)$.

Because we suspected the JCS score to be the most important confounder, we also conducted stratified analyses by JCS 0, 1-digit, 2-digit, and 3-digit codes (Supplementary Tables S2 and 3). Although coiling did not have a significant effect on discharge mRS scores of 3-6 in patients with JCS 0, 1-digit, and 2-digit codes, rates of discharge mRS scores of 3-6 in the coiling group were significantly lower in patients with 3 -digit codes in both the full cohort (OR 0.74, $\mathrm{p}=0.038$ ) and the subgroup with CSC scores $(\mathrm{OR} 0.70, \mathrm{p}=0.024)$. Although coiling did not have a significant effect on cerebral infarction in patients with JCS 0,1-digit, and 2-digit codes, rates of cerebral infarction in the coiling group were significantly higher in patients with 3 -digit codes in both the full cohort (OR 1.57, $\mathrm{p}=0.008)$ and the subgroup with CSC scores (OR 1.69, $\mathrm{p}=0.006$ ). Regarding in-hospital mortality, PSIs $\geq 1$, HACs $\geq 1$, hospital stay, and medical costs, the influence of treatment modality seemed similar between the clipping and coiling groups in patients with each JCS score.

\section{Propensity Score Analysis}

Following 1:1 matching, 1556 patients who had undergone clipping and 1556 who had undergone coiling fol-

TABLE 3. Outcomes of patients with SAH according to mixed-model analysis

\begin{tabular}{|c|c|c|c|c|c|c|}
\hline \multirow[b]{2}{*}{ Parameter } & \multicolumn{3}{|c|}{ Full Cohort } & \multicolumn{3}{|c|}{ Subgroup w/ CSC Scores } \\
\hline & $\mathrm{OR}^{*}$ & $95 \% \mathrm{Cl}$ & $p$ Value & $\mathrm{OR}^{*}$ & $95 \% \mathrm{Cl}$ & $\mathrm{p}$ Value \\
\hline In-hospital mortality & 1.3 & $1.06-1.59$ & 0.013 & 1.27 & $1.01-1.60$ & 0.046 \\
\hline Discharge mRS Scores 3-6 & 0.98 & $0.85-1.14$ & 0.761 & 0.96 & $0.81-1.14$ & 0.657 \\
\hline Cerebral infarction & 1.19 & $0.99-1.43$ & 0.066 & 1.18 & $0.96-1.45$ & 0.124 \\
\hline PSIs $\geq 1$ & 1.3 & $1.10-1.53$ & 0.003 & 1.33 & $1.10-1.60$ & 0.003 \\
\hline $\mathrm{HACs} \geq 1$ & 0.73 & $0.55-0.95$ & 0.021 & 0.78 & $0.58-1.05$ & 0.105 \\
\hline Length of hospital stay & $-7.18^{*} \dagger$ & -9.14 to -5.21 & $<0.001$ & $-6.98^{*} \dagger$ & -9.15 to -4.80 & $<0.001$ \\
\hline Medical costs & $-144.64^{*} \dagger$ & -1008.17 to 718.88 & 0.742 & $-603.15^{*} \dagger$ & -1546.05 to 339.75 & 0.21 \\
\hline
\end{tabular}

* Odds ratios and differences were adjusted by a mixed model with sex, age, JCS scores, and CCI scores as fixed effects and hospital as a random effect. Clipping was used as the reference.

$\dagger$ Number represents the $\beta$ value. 
lowing aneurysm rupture were matched based on demographic similarities. All covariates between the clipping and coiling groups were statistically indistinguishable after matching. Outcomes for patients with SAH after matching according to propensity score are shown in Table 4. In-hospital mortality was significantly higher in the coiling group (OR 1.25, $\mathrm{p}=0.047$ ). Rates of cerebral infarction (OR 0.97, $\mathrm{p}=0.745)$ and $\mathrm{mRS}$ scores of 3-6 at discharge (OR 0.94, $\mathrm{p}=0.409$ ) were not significantly different between the groups. The proportion of PSIs $\geq 1$ was significantly higher in the coiling group (OR 1.77, $\mathrm{p}<$ 0.001 ), whereas that of $\mathrm{HACs} \geq 1$ was not significantly different between the groups (OR 0.83, $\mathrm{p}=0.16)$. The median hospital stay was significantly longer in the clipping group $(\beta=-6.71, p<0.001)$, whereas the medical costs were not significantly different between the groups $(\beta=754.04, \mathrm{p}$ $=0.086)$. Compared with the results of the mixed-model analysis, except for the proportion of HACs $\geq 1$, a similar tendency was observed for the odds ratios and differences for the other factors.

\section{Validation of Diagnosis, Treatment Modality, and Outcomes}

Our sample, selected using 2-stage random sampling, consisted of 495 patients at 68 institutions. The sensitivity, specificity, and PPV of SAH as the primary diagnosis were $100 \%, 99.0 \%$, and $95.1 \%$, respectively. Among the 495 patients, 82 were identified using the ICD-10 diagnosis codes related to SAH (I60.0-9). The sensitivities, specificities, and PPVs of treatment modality and in-hospital mortality were all $100 \%$. The sensitivity, specificity, and PPV of mRS Scores 3-6 at discharge were 97.2\%, 87.0\%, and $85.4 \%$, respectively. The $\kappa$ coefficient of the JCS score on admission was 0.683 . The results of the validation study are summarized in Table 5.

\section{Discussion}

In Japan, the incidence of SAH is higher, ${ }^{8}$ and the associated mortality is lower, ${ }^{24}$ than in other countries, possibly because of differences in genetic backgrounds, socioeconomic indicators, ${ }^{13}$ and national medical systems, including centralization of neurosurgical and acute stroke care capabilities. Therefore, international, national, and

TABLE 4. Outcomes of patients with SAH according to propensity score analysis

\begin{tabular}{lccr}
\hline \multicolumn{1}{c}{ Parameter } & $\mathrm{OR}^{*}$ & $95 \% \mathrm{Cl}$ & $\mathrm{p} \mathrm{Value}$ \\
\hline In-hospital mortality & 1.25 & $1.00-1.57$ & 0.047 \\
\hline Discharge mRS Scores $3-6$ & 0.94 & $0.82-1.08$ & 0.409 \\
\hline Cerebral infarction & 0.97 & $0.81-1.16$ & 0.745 \\
\hline PSIs $\geq 1$ & 1.77 & $1.53-2.04$ & $<0.001$ \\
\hline HACs $\geq 1$ & 0.83 & $0.64-1.08$ & 0.16 \\
\hline Length of hospital stay & $-6.70^{*} \dagger$ & -8.64 to -4.77 & $<0.001$ \\
\hline Medical costs & $754.04^{*} \dagger$ & -106.89 to 1614.96 & 0.086 \\
\hline
\end{tabular}

* Odds ratios and differences were estimated for outcomes after 1:1 propensity score matching to account for differences in baseline characteristics between groups. Clipping was used as the reference.

† Number represents the $\beta$ value.
TABLE 5. Results of the validation study

\begin{tabular}{lccc}
\hline \multicolumn{1}{c}{ Parameter } & Sensitivity & Specificity & PPV \\
\hline SAH as primary diagnosis & $100 \%$ & $99.0 \%$ & $95.1 \%$ \\
\hline Treatment modality & $100 \%$ & $100 \%$ & $100 \%$ \\
\hline In-hospital mortality & $100 \%$ & $100 \%$ & $100 \%$ \\
\hline Discharge mRS Scores 3-6 & $97.2 \%$ & $87.0 \%$ & $85.4 \%$ \\
\hline
\end{tabular}

longitudinal comparisons of outcomes for patients with SAH treated using different therapeutic modalities should be discussed in light of socioeconomic indicators, acute stroke care capabilities at the specific time, $, 13,14,26,28,31$ and patient-level characteristics such as SAH severity. ${ }^{27}$ The present study is the first to compare outcomes for SAH patients treated with clipping and coiling via a mixed-model analysis of a large nationwide discharge database (DPC database) after adjustment for patient-level characteristics including age, sex, level of consciousness (JCS score), CCI score, and hospital-level CSC capabilities (CSC score). ${ }^{14,15}$ Our results revealed that patients who had undergone coiling exhibited significantly higher in-hospital mortality $(12.4 \%$ vs $8.7 \%$ ) and shorter hospital stays (32.0 vs 37.0 days) than those in patients who had undergone clipping. The proportion of patients discharged with mRS scores of 3-6 (coiling 46.4\%, clipping 42.9\%) and median medical costs (thousands US\$: coiling 35.7, clipping 36.7) were not significantly different between the groups. Furthermore, the high accuracy of diagnoses, treatment modalities, and outcomes of the DPC cohort in the present study were confirmed using a 2-stage sampling method.

\section{Discrepancies in the Results of Comparative Studies}

Our results were partly consistent with those of 2 previous RCTs - the ISAT and the Barrow Ruptured Aneurysm Trial (BRAT) - with regard to functional outcomes, although rates of in-hospital mortality differed significantly. ${ }^{18,20}$ One possible explanation for this discrepancy is that almost $80 \%$ of all eligible aneurysms were excluded from the ISAT cohort, calling into question the general applicability of the results. In the BRAT, a relatively small number of patients were randomized and treated by highly experienced surgeons and endovascular physicians at a single high-volume center. The merits of using an electronic hospital claim database are associated with decreases in the risk of selection biases. Such biases may largely account for the differences between results of the present study and those of previous RCTs.

Researchers have increasingly relied on administrative databases of hospital admissions to assess clinical outcomes in patients with SAH. Three nationwide database studies were conducted to compare outcomes for patients with SAH who had undergone clipping or coiling, yielding mixed results., ${ }^{7,26}$ Using administrative claim data from Canada entered between 1995 and 2004, authors of one study found that endovascular coiling was associated with a significantly increased risk of death or SAH readmission. ${ }^{26}$ The results were confirmed by propensity scorematched analysis. Authors of another study, who used the National (Nationwide) Inpatient Sample from 1998 to 2003 in the US, reported higher in-hospital mortality after coil- 
ing than after clipping (16.6\% vs $12.7 \%)^{7}$ These 2 studies, both conducted relatively soon after the publication of the ISAT, produced results similar to ours in terms of the case volume ratio, mortality, and functional outcomes of clipping and coiling. ${ }^{7,26}$ Qureshi et al. revealed that, after the publication of the ISAT in 2002, there was a significant increase in endovascular treatment as well as a decrease in in-hospital mortality in patients with $\mathrm{SAH} .{ }^{28} \mathrm{~A}$ third nationwide database study used the Premier database, which is a voluntary, fee-supported collection of data developed to assess the quality and resource utilization of health care delivery in the US. ${ }^{17}$ In contrast to findings in the present and aforementioned studies, the Premier database study revealed no significant differences in mortality between the groups (clipping 12\%, coiling 13\%, OR 0.94, 95\% CI 0.731.21), though functional outcomes were significantly better in the coiling group (discharge to long-term care: clipping $42 \%$, coiling 36\%). ${ }^{17}$ Except for the present study, the only other study to use an administrative database in the analysis of patients treated relatively late after the publication of the ISAT was a US study in which patients admitted from 2006 to 2011 demonstrated higher in-hospital mortality after clipping (11.9\% vs $8.7 \%$ ) and similar in-hospital mortality after coiling (12.6\% vs $12.5 \%$ ), relative to patients in the present study ${ }^{17}$ Since age is a predictor of a poor outcome,, 24 it is important to note that the patients with SAH who had undergone clipping (mean age 62.0 years) and coiling (63.5 years) in our Japanese cohort were almost 10 years older than those in the US and Canadian studies., 17,26 Nonetheless, in-hospital mortality rates after clipping were much lower in Japan than the corresponding rates in the other countries, whereas rates after coiling remained similar.

\section{Unmeasured Confounders}

Discrepancies between findings of the present and previous large-scale database studies may be explained by the influence of unmeasured confounders. In general, the most common cause of death and poor functional outcomes following SAH is the initial bleeding. ${ }^{5,16} \mathrm{In}$ fact, we revealed that the influence of treatment modalities on rates of discharge mRS scores of 3-6 and cerebral infarction differs between patients with 0 to 2-digit JCS scores and those with 3-digit JCS scores. Although McDonald et al. performed propensity score analysis to minimize potential selection bias, SAH severity was not included in the patient variables used to generate propensity scores, making interpretation of the results difficult. ${ }^{17}$ On the other hand, SAH severity was adjusted by the JCS score ${ }^{14,25,29}$ in the present study, minimizing its confounding effects. Notably, the importance of the JCS, first developed in 1974 for the prediction of stroke outcomes, has recently been validated. ${ }^{29}$ The second most common cause of SAH-related death and poor functional outcome is rebleeding. ${ }^{5,16}$ Earlier treatment of ruptured aneurysms reduces the incidence of rebleeding. ${ }^{33}$ However, no significant difference in the mean preoperative period ( 2 days) between clipping and coiling was observed in the present study. Intracranial aneurysm size and location are also important clinical variables used to guide treatment decisions. However, details regarding these variables are unavailable in administrative databases and represent potentially important unmeasured confounders. In this regard, the Prospective Registry of Subarachnoid Aneurysms Treatment (PRESAT) trial provided reasonable estimates of the differences in ruptured aneurysm size and location in patients treated with clipping versus coiling. ${ }^{31}$ The PRESAT trial was undertaken from 2006 to 2007 at 29 tertiary care referral centers in Japan where both clipping and coiling were available for ruptured cerebral aneurysms, and the treatment choice was based on both patient and aneurysm characteristics. ${ }^{31}$ Clipping was mostly performed for small aneurysms with wide necks and for middle cerebral artery aneurysms, whereas coiling was preferred for larger, internal carotid artery and posterior circulation aneurysms. Authors of the study concluded that treatment modality, aneurysm size, and aneurysm location did not influence outcomes at tertiary care referral hospitals with high CSC capabilities.

Ideally, ruptured cerebral aneurysms should be treated at centers offering high-quality treatment with open surgical procedures as well as endovascular techniques. Our study demonstrated that high-quality surgical clipping remains available as both a first-line and a complementary treatment option for ruptured cerebral aneurysms in Japan: Our results were similar regardless of whether total CSC scores were adjusted in the mixed-model analysis. The optimal balance between clipping and coiling to maximize the overall proportion of favorable outcomes in patients with SAH will vary somewhat among centers, regions, and countries, depending on local expertise and CSC capabilities.

After CSC capabilities (total CSC scores) were adjusted in the mixed model, the odds ratios of in-hospital mortality and unfavorable outcomes of coiling compared with clipping remained almost unchanged. Although coiling was performed in hospitals with significantly higher CSC scores, the effect of treatment modality on outcomes in patients with SAH was unaffected by CSC capabilities. This finding is unexpected since higher CSC capabilities were found to be significantly associated with reduced inhospital mortality in patients with SAH in our previous analysis of the J-ASPECT study. ${ }^{14}$ Rather than the CSC capabilities, surgeon-related factors such as technical skill and patient-related factors such as aneurysm location, size, and morphology may primarily influence the association between treatment modality and outcome. Further study is required to assess the influence of surgical skill and aneurysm characteristics.

In Japan, the official designation of primary stroke centers or CSCs has not yet begun, although the clear correlation between comprehensive stroke care capabilities and in-hospital mortality for all types of stroke revealed using the J-ASPECT Study database has had a significant impact on national policy. Given recently established evidence for the efficacy of mechanical thrombectomy in patients with acute ischemic stroke, a task force headed by the Japan Stroke Society and the Japan Neurosurgical Society has initiated an active discussion regarding the recommended items and criteria for the designation of primary stroke centers and CSCs in Japan, such as the CSC score. ${ }^{14}$

\section{Study Strengths and Limitations}

In light of the possible selection bias, the present study 
can be considered a snapshot of the state of ruptured aneurysm treatment in Japan rather than a direct comparison between the safety and efficacy of both treatments. Given that heterogeneity in patient outcomes following stroke has been observed across different hospitals in the same country, ${ }^{14}$ considering the patients within each hospital as a cluster in a mixed model may be superior to conventional regression, which views all participants as independent. The present study is the first in which outcomes in patients with SAH treated with clipping and coiling have been compared using a mixed-model analysis of a large nationwide discharge database (DPC database), after adjustment for important patient and hospital characteristics. ${ }^{14,15}$ In addition, we added a propensity score analysis to verify the results of the mixed-model analysis. Findings of the propensity score analysis were mostly consistent with those of the mixed-model analysis. Therefore, we consider the results of our study to be highly reliable.

Our study has several limitations. First, the DPC database lacks several types of data such as cause of death and aneurysm characteristics. As previously mentioned, such confounders peculiar to Japan may underlie the differences in outcomes compared with other countries. Second, as a measure of SAH severity, we used the JCS score rather than the Hunt and Hess grade or the World Federation of Neurological Surgeons (WFNS) grade. The JCS is widely used for the assessment of impaired consciousness in patients with SAH in Japan., ${ }^{2,12,14,25,32}$ Although the JCS cannot assess neurological impairment other than diminished consciousness, the JCS score on admission had the greatest impact on the outcomes of patients with SAH. ${ }^{9}$ In addition, JCS scores have been reported to correlate with stroke outcomes. ${ }^{29}$ Therefore, we believe that the JCS is sufficient for clinical grade assessment in patients with SAH. However, as an increasing number of stroke researchers have used JCS scores in recent Japanese DPC database studies, $2,9,12,14,25,30,33$ it may be necessary to compare the JCS scoring system with more typical grading scales. Third, the DPC database includes information collected only during hospitalization; thus, we were unable to evaluate long-term outcomes. However, the ISAT reported that the probability of death or dependency at 2 months, 1 year, and 10 years after treatment were higher in the clipping group. ${ }^{19,20}$ Results of the ISAT suggest that short-term outcomes regarding mRS scores and mortality may serve as reasonable predictive markers for long-term outcomes. Fourth, the external validity of the CSC grading system has not been confirmed. External validation is necessary to increase the reliability of self-assessment; therefore, we aim to validate information regarding hospital characteristics and outcomes using a small sample set from the validation cohort of the present study.

\section{Conclusions}

Clipping remains the standard treatment for SAH in Japan. Compared with coiling, clipping was associated with reduced in-hospital mortality, longer hospital stay, and similar unfavorable functional outcomes and medical costs in Japan in 2012. These findings were independent of CSC capabilities. Further study is required to determine the effects of unmeasured confounders on the outcomes of clipping and coiling.

\section{Acknowledgments}

We thank the J-ASPECT study collaborators for their important contributions. The names of the 393 participating hospitals and representatives are listed in the online-only data supplement. We also thank Drs. Manabu Hasegawa, Tomoatsu Tsuji, and Yasuhiro Nishijima for their helpful discussions; Profs. Takamasa Kayama and Nobuo Hashimoto for their supervision of the collaboration with the Japan Neurosurgical Society; and Ms. Arisa Ishitoko for her secretarial assistance.

This work was supported by Grants-in-Aid from the Ministry of Health, Labor, and Welfare of Japan and JSPS KAKENHI Grant No. 25293314 (K.I.). This research is partially supported by the Practical Research Project for Life-Style Related Diseases including Cardiovascular Diseases and Diabetes Mellitus from Japan Agency for Medical Research and development, AMED.

\section{References}

1. Alberts MJ, Latchaw RE, Selman WR, Shephard T, Hadley MN, Brass LM, et al: Recommendations for comprehensive stroke centers: a consensus statement from the Brain Attack Coalition. Stroke 36:1597-1616, 2005

2. Asano S, Hara T, Haisa T, Okamoto K, Kato T, Ohno H, et al: Outcomes of 24 patients with subarachnoid hemorrhage aged 80 years or older in a single center. Clin Neurol Neurosurg 109:853-857, 2007

3. Berman MF, Solomon RA, Mayer SA, Johnston SC, Yung PP: Impact of hospital-related factors on outcome after treatment of cerebral aneurysms. Stroke 34:2200-2207, 2003

4. Brinkman GL, Coates EO Jr: The effect of bronchitis, smoking, and occupation on ventilation. Am Rev Respir Dis 87:684-693, 1963

5. Broderick JP, Brott TG, Duldner JE, Tomsick T, Leach A: Initial and recurrent bleeding are the major causes of death following subarachnoid hemorrhage. Stroke 25:1342-1347, 1994

6. Charlson ME, Pompei P, Ales KL, MacKenzie CR: A new method of classifying prognostic comorbidity in longitudinal studies: development and validation. J Chronic Dis 40:373383, 1987

7. Cowan JA Jr, Ziewacz J, Dimick JB, Upchurch GR Jr, Thompson BG: Use of endovascular coil embolization and surgical clip occlusion for cerebral artery aneurysms. J Neurosurg 107:530-535, 2007

8. de Rooij NK, Linn FH, van der Plas JA, Algra A, Rinkel GJ: Incidence of subarachnoid haemorrhage: a systematic review with emphasis on region, age, gender and time trends. J Neurol Neurosurg Psychiatry 78:1365-1372, 2007

9. Deruty R, Pelissou-Guyotat I, Mottolese C, Amat D, Bognar $\mathrm{L}$ : Level of consciousness and age as prognostic factors in aneurysmal SAH. Acta Neurochir (Wien) 132:1-8, 1995

10. Fargen KM, Neal D, Rahman M, Hoh BL: The prevalence of patient safety indicators and hospital-acquired conditions in patients with ruptured cerebral aneurysms: establishing standard performance measures using the Nationwide Inpatient Sample database. J Neurosurg 119:1633-1640, 2013

11. Fonarow GC, Zhao X, Smith EE, Saver JL, Reeves MJ, Bhatt DL, et al: Door-to-needle times for tissue plasminogen activator administration and clinical outcomes in acute ischemic stroke before and after a quality improvement initiative. JAMA 311:1632-1640, 2014

12. Gotoh O, Tamura A, Yasui N, Nihei H, Manaka S, Suzuki A, et al: [Japan Coma Scale in the prediction of outcome after early surgery for aneurysmal subarachnoid hemorrhage.] No To Shinkei 47:49-55, 1995 (Jpn) 
13. Guha D, Ibrahim GM, Kertzer JD, Macdonald RL: National socioeconomic indicators are associated with outcomes after aneurysmal subarachnoid hemorrhage: a hierarchical mixedeffects analysis. J Neurosurg 121:1039-1047, 2014

14. Iihara K, Nishimura K, Kada A, Nakagawara J, Ogasawara $\mathrm{K}$, Ono J, et al: Effects of comprehensive stroke care capabilities on in-hospital mortality of patients with ischemic and hemorrhagic stroke: J-ASPECT study. PLoS One 9:e96819, 2014

15. Iihara K, Nishimura K, Kada A, Nakagawara J, Toyoda K, Ogasawara K, et al: The impact of comprehensive stroke care capacity on the hospital volume of stroke interventions: a nationwide study in Japan: J-ASPECT study. J Stroke Cerebrovasc Dis 23:1001-1018, 2014

16. Le Roux PD, Elliott JP, Newell DW, Grady MS, Winn HR: Predicting outcome in poor-grade patients with subarachnoid hemorrhage: a retrospective review of 159 aggressively managed cases. J Neurosurg 85:39-49, 1996

17. McDonald JS, McDonald RJ, Fan J, Kallmes DF, Lanzino G, Cloft HJ: Comparative effectiveness of ruptured cerebral aneurysm therapies: propensity score analysis of clipping versus coiling. AJNR Am J Neuroradiol 35:164-169, 2014

18. McDougall CG, Spetzler RF, Zabramski JM, Partovi S, Hills NK, Nakaji P, et al: The Barrow Ruptured Aneurysm Trial. J Neurosurg 116:135-144, 2012

19. Molyneux AJ, Birks J, Clarke A, Sneade M, Kerr RS: The durability of endovascular coiling versus neurosurgical clipping of ruptured cerebral aneurysms: 18 year follow-up of the UK cohort of the International Subarachnoid Aneurysm Trial (ISAT). Lancet 385:691-697, 2015 (Erratum in Lancet 385:946, 2015)

20. Molyneux A, Kerr R, Stratton I, Sandercock P, Clarke M, Shrimpton J, et al: International Subarachnoid Aneurysm Trial (ISAT) of neurosurgical clipping versus endovascular coiling in 2143 patients with ruptured intracranial aneurysms: a randomised trial. Lancet 360:1267-1274, 2002

21. Molyneux AJ, Kerr RS, Yu LM, Clarke M, Sneade M, Yarnold JA, et al: International subarachnoid aneurysm trial (ISAT) of neurosurgical clipping versus endovascular coiling in 2143 patients with ruptured intracranial aneurysms: a randomised comparison of effects on survival, dependency, seizures, rebleeding, subgroups, and aneurysm occlusion. Lancet 366:809-817, 2005

22. Nakamura K: Diagnosis procedure combination database would develop nationwide clinical research in Japan. Circ J 80:2289-2290, 2016

23. Nichols DA, Brown RD Jr, Meyer FB: Coils or clips in subarachnoid haemorrhage? Lancet 360:1262-1263, 2002

24. Nieuwkamp DJ, Setz LE, Algra A, Linn FH, de Rooij NK, Rinkel GJ: Changes in case fatality of aneurysmal subarachnoid haemorrhage over time, according to age, sex, and region: a meta-analysis. Lancet Neurol 8:635-642, 2009

25. Ohta T, Kikuchi H, Hashi K, Kudo Y: Nizofenone administration in the acute stage following subarachnoid hemorrhage. Results of a multi-center controlled double-blind clinical study. J Neurosurg 64:420-426, 1986

26. O'Kelly CJ, Kulkarni AV, Austin PC, Wallace MC, Urbach D: The impact of therapeutic modality on outcomes following repair of ruptured intracranial aneurysms: an administrative data analysis. Clinical article. J Neurosurg 113:795801,2010

27. Pegoli M, Mandrekar J, Rabinstein AA, Lanzino G: Predictors of excellent functional outcome in aneurysmal subarachnoid hemorrhage. J Neurosurg 122:414-418, 2015

28. Qureshi AI, Vazquez G, Tariq N, Suri MF, Lakshminarayan K, Lanzino G: Impact of International Subarachnoid An- eurysm Trial results on treatment of ruptured intracranial aneurysms in the United States. Clinical article. J Neurosurg 114:834-841, 2011

29. Shigematsu K, Nakano H, Watanabe Y: The eye response test alone is sufficient to predict stroke outcome-reintroduction of Japan Coma Scale: a cohort study. BMJ Open 3:e002736, 2013

30. Spetzler RF, McDougall CG, Albuquerque FC, Zabramski JM, Hills NK, Partovi S, et al: The Barrow Ruptured Aneurysm Trial: 3-year results. J Neurosurg 119:146-157, 2013

31. Taki W, Sakai N, Suzuki H: Determinants of poor outcome after aneurysmal subarachnoid hemorrhage when both clipping and coiling are available: Prospective Registry of Subarachnoid Aneurysms Treatment (PRESAT) in Japan. World Neurosurg 76:437-445, 2011

32. Todo T, Usui M, Takakura K: Treatment of severe intraventricular hemorrhage by intraventricular infusion of urokinase. J Neurosurg 74:81-86, 1991

33. Whitfield PC, Moss H, O'Hare D, Smielewski P, Pickard JD, Kirkpatrick PJ: An audit of aneurysmal subarachnoid haemorrhage: earlier resuscitation and surgery reduces inpatient stay and deaths from rebleeding. J Neurol Neurosurg Psychiatry 60:301-306, 1996

34. Yasunaga H, Ide H, Imamura T, Ohe K: Impact of the Japanese Diagnosis Procedure Combination-based Payment System on cardiovascular medicine-related costs. Int Heart J 46:855-866, 2005

35. Yasunaga H, Matsui H, Horiguchi H, Fushimi K, Matsuda S: [Application of the diagnosis procedure combination (DPC) data to clinical studies.] J UOEH 36:191-197, 2014 (Jpn)

\section{Disclosures}

Dr. Yoshimura has participated in the speaker's bureau and/or has received honoraria from Bayer, Sanofi, Boehringer-Ingelheim, and Otsuka Pharmaceutical Co. and has received grants from Terumo and Takeda Pharmaceutical Co.

\section{Author Contributions}

Conception and design: Iihara. Acquisition of data: Iihara, Nakagawara, Toyoda, Ogasawara, Ono, Shiokawa, Aruga, Miyachi, Nagata, Matsuda, Yoshimura, Okuchi, Suzuki, Nakamura. Analysis and interpretation of data: all authors. Drafting the article: Kurogi. Critically revising the article: Iihara. Reviewed submitted version of manuscript: Kurogi, A Nishimura, Sayama, Nakagawara, Toyoda, Ogasawara, Ono, Shiokawa, Aruga, Miyachi, Nagata, Matsuda, Yoshimura, Okuchi, Suzuki, Nakamura, Onozuka, Hagihara. Statistical analysis: K Nishimura, Kamitani, Nakamura, Onozuka, Hagihara. Administrative/technical/material support: K Nishimura, Kamitani. Study supervision: Iihara.

\section{Supplemental Information Online-Only Content}

Supplemental material is available with the online version of the article.

Online Supplement. https://thejns.org/doi/suppl/10.3171/2016. 12.JNS161039.

\section{Correspondence}

Koji Iihara, Department of Neurosurgery, Graduate School of Medical Sciences, Kyushu University, 3-1-1, Maidashi, Higashiku, Fukuoka 812-8582, Japan. email: kiihara@ns.med.kyushu-u. ac.jp. 